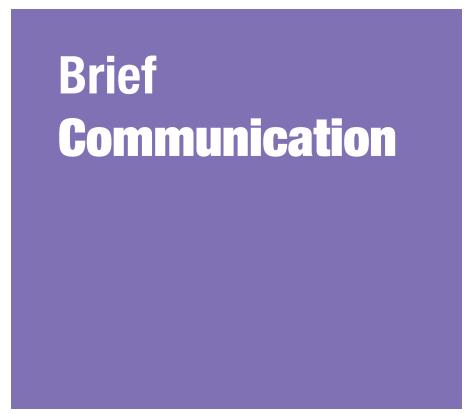

Submitted: 28 June 2016 Accepted: 26 Sept 2016 Online: 7 Dec 2016

\section{Characteristics and Outcomes of Patients with Anterior Circulation Intracranial Aneurysm Managed with Clipping in Hospital Sungai Buloh}

\author{
Allani Ab. Ghani ${ }^{1,2,3,4}$, Saiful Azl Mat Nayan', Regunath \\ Kandasamy ${ }^{3}$, Azmin Kass Rosman ${ }^{1}$, Abdul Rahman Izanı Ghani ${ }^{3}$
}

\author{
1 Department of Neurosurgery, Hospital Sungai Buloh, Jalan Hospital, \\ 47000 Sungai Buloh, Selangor, Malaysia \\ 2 Center for Neuroscience Services and Research, Universiti Sains Malaysia, \\ Jalan Sultanah Zainab 2, 16150 Kubang Kerian, Kelantan, Malaysia \\ 3 Department of Neurosciences, Hospital Universiti Sains Malaysia, Jalan \\ Hospital USM, 16150 Kubang Kerian, Kelantan, Malaysia \\ 4 Department of Neurosciences, Universiti Sains Malaysia, Jalan Sultanah \\ Zainab 2, 16150 Kubang Kerian, Kelantan, Malaysia
}

To cite this article: Ailani AG, Saiful Azli MN, Regunath K, Azmin Kass R, Abdul Rahman Izani G. Characteristics and outcomes of patients with anterior circulation intracranial aneurysm managed with clipping in Hospital Sungai Buloh. Malays J Med Sci. 2016;23(6):113-117. http://dx.doi.org/10.21315/mjms2016.23.6.12

To link to this article: http://dx.doi.org/10.21315/mjms2016.23.6.12

\title{
Abstract
}

Introduction: The annual incidence of intracranial aneurysm in Malaysia is estimated to be 1.1-1.7 per 100,000 population based on a study done conducted in 1988. Since then, little epidemiological research has been conducted in Malaysia, and the real incidence is therefore probably unreported despite advancements in the diagnosis and treatment of this disorder. Intracranial aneurysm may be treated by microsurgical clipping or embolisation depending on its location and the surgeon's preference. This study aims to report the characteristics and outcomes of patients with a clipped anterior circulation aneurysm in Hospital Sungai Buloh.

Methods: We retrospectively collected the data of patients with anterior circulation aneurysms who underwent clipping from 1 January 2013 until 30 June 2014 in Hospital Sungai Buloh.

Results: The mean age of the patients was 48.9 years old, and $56.7 \%$ of the study population were male and $63.3 \%$ were Malay. There were almost equal numbers of patients with (46.7\%) and without (53.3\%) co-morbidities such as hypertension and ischaemic heart disease. Half of the study population fell under Fisher grade 3 (50.0\%), whereas $46.7 \%$ were Fisher grade 4. With respect to the Navarro score, $20 \%$ of patients scored $10,16.7 \%$ scored 5 or $7,13.3 \%$ scored $11,10 \%$ scored $9,6.7 \%$ scored 1 and $3.3 \%$ scored 2,3 or 8 . Most of the patients fell under grade I (33.3\%) of the World Federation of Neurological Societies grading (WFNS), and the fewest number of patients were grade III (3.3\%). Most (56.7\%) patients had an intracranial aneurysm located at the anterior communicating artery (ACOM), followed by the anterior cerebral artery (ACA) and the middle cerebral artery (MCA) (16.7\% each), the posterior communicating artery (PCOM) (6.7\%) and, finally, the internal carotid artery (ICA) (3.3\%). Neurological outcomes at three and six months were assessed using the Modified Rankin Scale (mRS). At three months follow-up, the majority of the participants (33.3\%) scored o or 6 , whereas at six months follow-up, the majority scored o (43.3\%).

Conclusion: The main characteristics of patients in Hospital Sungai Buloh were that they were in their 40s and mostly Malays without any known comorbidities. Although the majority of patients had high Fisher grades, their outcomes post intervention were promising. However, 
as data collected from Hospital Sungai Buloh may not be representative of the whole country, a larger-scale data collection is necessary to create our own database and to identify the risks factors that are exclusive to our country, if present.

Keywords: intracranial aneurysm, subarachnoid hemorrhage, characteristic, outcome, Malaysia

\section{Background}

The incidence of ruptured intracranial aneurysm exhibits substantial geographic differences that reach 10 -fold among some countries (1). Malaysia and China have lower incidences compared to rates reported in Japan (22.7 per 100,000 population) and Finland (19.7 per 100,000 population) (2). To date, in Malaysia, there are only two datasets available regarding intracranial aneurysms: studies conducted by Chee and Loh in 1988 and Wong et al. in $2004(3,4)$. Few epidemiological studies are available on ruptured intracranial aneurysm compared to unruptured intracranial aneurysm because of the natural history of the disease, which has a high mortality rate once rupture occurs. Therefore, cases may go undetected and unrecorded. In general, the outcomes of patients with ruptured intracranial aneurysms who receive hospital care are still dismal, and the cost is expensive. The cost involved is directly related to the treatment, and affected patients are usually within the productive age group. Successfully treated patients may also succumb to death later from vasospasm or its complications. The purpose of this study is to create our own database for cases of anterior circulation intracranial aneurysm managed in Hospital Sungai Buloh from 1 January 2013 to 30 June 2014, including the outcomes of these patients.

\section{Methods}

A total of 30 patients with a clipped anterior circulation intracranial aneurysm managed in Hospital Sungai Buloh between 1 Jan 2013 and 30 June 2014 were included in this study. Patient data were collected from the hospital's electronic database. Data collected include the patient's admission demographic profile, Fisher, Navarro, WFNS score and the modified Rankin score (mRS) at three and six months after discharge. Other data include patient comorbidities and the location of the aneurysm.

\section{Results}

The mean age of the patients was 48.9 years old. The majority of the patients $(46.7 \%)$ were 40 to 49 years old, followed by $20.0 \%$ aged 30 to 39 or over 60 years of age. The smallest (13.3\%) number of patients was aged 50 to 59 . More than half of the study population were male $(56.7 \%)$ and of Malay ethnicity (63.3\%). Refer Table 1 for description of study participants.

Table 1. Description of study participants

\begin{tabular}{lcc} 
Characteristics & & \\
Age & Mean (SD) & $48.90(12.26)$ \\
& $30-39$ & $6(20.0)$ \\
& $40-49$ & $14(46.7)$ \\
& $50-59$ & $4(13.3)$ \\
& $>60$ & $6(20.0)$ \\
Gender & Male & $17(56.7)$ \\
& Female & $13(43.3)$ \\
Ethnicity & Malay & $19(63.3)$ \\
& Chinese & $8(26.7)$ \\
& Indian & $1(3.3)$ \\
& Others & $2(6.7)$ \\
\hline
\end{tabular}

Data presented as $n(\%)$ unless indicated

As tabulated in Table 2, there were almost equal numbers of patients with (46.7\%) and without (53.3\%) co-morbidities. Among the 14 patients with co-morbidities, 12 had hypertension, six had diabetes and three had other co-morbidities, such as bronchial asthma and ischaemic heart disease.

The presenting Fisher, Navarro and WFNS grade are as tabulated in Table 3. Half of the study population fell under Fisher grade 3 (50.0\%), whereas $46.7 \%$ were Fisher grade 4. With respect to the Navarro score, $20 \%$ of patients scored 10, $16.7 \%$ scored 5 or $7,13.3 \%$ scored $11,10 \%$ scored $9,6.7 \%$ scored 1 and $3.3 \%$ scored 2, 3 or 8. Most patients fell under grade I (33.3\%) of the World Federation of Neurological Societies grading (WFNS), followed by grade $\mathrm{V}$ (26.7\%), grade IV (20.0\%), grade II (16.7\%) and grade III (3.3\%). 
Table 2. Presence of co-morbidities in study participants

\begin{tabular}{lcc} 
& & $n(\%)$ \\
Co-morbidities & Yes & $14(46.7)$ \\
& No & $16(53.3)$ \\
Hypertension $^{\mathrm{a}}$ & Yes & $12(85.7)$ \\
& No & $2(14.3)$ \\
Diabetes $^{\mathrm{a}}$ & Yes & $6(42.9)$ \\
Other co-morbidities $^{\mathrm{a}, \mathrm{b}}$ & No & $8(51.7)$ \\
& Yes & $3(21.4)$ \\
& No & $11(78.6)$ \\
\hline
\end{tabular}

aPresented as per within group of patients with co-morbidities ${ }^{\mathrm{b}}$ Athma and IHD

Table 3. Fisher, WFNS and Navarro score of study participants

\begin{tabular}{lcc} 
& Scores & $n(\%)$ \\
Fisher & 0 & $1(3.3)$ \\
& 3 & $15(50.0)$ \\
Navarro & 4 & $14(46.7)$ \\
& 1 & $2(6.7)$ \\
& 2 & $1(3.3)$ \\
& 3 & $1(3.3)$ \\
& 4 & $0(0)$ \\
& 5 & $5(16.7)$ \\
6 & $2(6.7)$ \\
7 & $5(16.7)$ \\
8 & $1(3.3)$ \\
WFNS & 9 & $3(10)$ \\
& 10 & $6(20)$ \\
& 11 & $4(13.3)$ \\
& I & $10(33.3)$ \\
II & $5(16.7)$ \\
III & $1(3.3)$ \\
IV & $6(20.0)$ \\
V & $8(26.7)$ \\
\hline
\end{tabular}

More than half of the patients' intracranial aneurysms were located at the anterior communicating artery (ACOM) (56.7\%), followed by the anterior cerebral artery (ACA) and middle cerebral artery (MCA) (16.7\% each), the posterior communicating artery (PCOM) (6.7\%) and finally the internal carotid artery (ICA) (3.3\%).

The neurological outcomes at three and six months were assessed using the Modified Rankin Scale (mRS) as in Table 4. At three months follow-up, the majority of the participants
(33.3\%) scored o or 6 , followed by a score of $5(20.0 \%)$, a score of $1(6.7 \%)$, and $3.3 \%$ each for a score of 3 or 4 . At six months follow-up, the majority scored o (43.3\%), followed by 6 (33.3\%), 5 (20.0\%) and 4 (3.3\%).

Table 4. Modified Rankin Scale (mRS) of study participants at three and six months post clipping of intracranial aneurysm

\begin{tabular}{lcc} 
& & $n(\%)$ \\
mRS outcome at three months & 0 & $10(33.3)$ \\
& 1 & $2(6.7)$ \\
3 & $1(3.3)$ \\
4 & $1(3.3)$ \\
mRS outcome at six months & 5 & $6(20.0)$ \\
& 6 & $10(33.3)$ \\
& 4 & $13(43.3)$ \\
& 5 & $6(20.0)$ \\
& 6 & $10(33.3)$ \\
\hline
\end{tabular}

\section{Discussion}

This retrospective study analyses the data on the anterior circulation intracranial aneurysms that were clipped in Hospital Sungai Buloh between 1 January 2013 and 30 June 2014. The mean age of affected patients was 48.9 years old, and the majority of patients were of Malay descent (63.3\%) and male (56.7\%). Of the patients, $53.3 \%$ did not have any comorbidities, $50 \%$ were Fisher 3, had a Navarro score of 10 (20\%) and had good WFNS grade (WFNS I, $33.3 \%)$. The outcome of this clipped aneurysm is good based on the good mRS at both three and six months after discharge.

According to Rinkel et al., the incidence of intracranial aneurysm increased with age, and the majority of cases occur among patients in their 50s (5). In this study, the mean age of the patients was younger at $48.90(\mathrm{SD}=12.26)$ years; with majority of the patients were between 40 and 49 years of age, and the least number of patients was between 50 and 59 years of age. There was also an equal percentage of patients in their 30 s and 6os. Male patients (56.7\%) were more frequent than females (43.3\%), which was in contrast to the usual pattern in which females are more affected than males (6). However, this ratio does not seem to be applicable in all populations and may vary from country to country (7). In this study, Malays 
(63.3\%) were more frequently affected than other races. However, this can be due to the racial distribution of Malaysia in general.

Although in this study $53.3 \%$ of patients did not have any known previous co-morbidities, of those who did have co-morbidity, $85.7 \%$ had hypertension. The variation of blood pressure and hypertension are known risk factors for the development of intracranial aneurysm (8). Intracranial arteries are composed of the outer layer of the adventitia, a muscular media that maintains most of the vessel wall integrity and the inner layer of intima. They are different from other vessels in the body as they do not possess external elastic lamina. Therefore, there is less elasticity of the media in addition to the thinner adventitia compared to the extracranial artery wall. These vessels are also situated in the subarachnoid space, which has no surrounding connective tissue to support the vessels (9). These factors make intracranial arteries susceptible to the formation of saccular aneurysms, making their occurrence fairly common in hypertensive patients and in patients with congenital conditions that predispose them to defects in the muscular layer of the arterial wall, such as autosomal dominant polycystic kidney disease, fibromuscular dysplasia, EhlerDanlos syndrome and Marfan syndrome (5, 10). Those patients without hypertension in this study might have not been diagnosed with hypertension previously. These findings emphasise the importance of hypertension screening and control.

Among the 30 patients who underwent clipping, the majority were Fisher $3(50 \%)$ or Fisher $4(46.7 \%)$, indicating that most patients only presented post intracranial aneurysm rupture with an additional risk of vasospasm. In addition, this also indicates that the incidental findings of intracranial aneurysm are still rare despite the improvement of Malaysia's health services.

Another study by Navarro adds up five factors (WFNS score, known case of hypertension, clot thickness, intraventricular haemorrhage and hydrocephalus on a CT of the brain) to correlate with angiographic vasospasm. According to Navarro, a score of 1-4 out of a total of 11 has $100 \%$ sensitivity but $8 \%$ specificity. A higher score has lower sensitivity but greater specificity (11). The scores range from $0-11$, and the patients are dispersed within that range; the highest, with a Navarro score of 10 (20\%), were followed by scores of 5 or 7 (both 16.7\%) and the lowest score of o (o\%).
The WFNS score is used to predict the outcome of intracranial aneurysm patients, although its specificity and sensitivity are questionable (12). In this study, 33.3\% of patients had a WFNS score of I, $26.7 \%$ had a WFNS score of $\mathrm{V}$, and the remaining patients fell between these scores. Therefore, we can predict that we would have similar percentage of actual outcomes, as the higher WFNS grades may indicate poorer outcomes. However, the scores are usually obtained during a patient's initial admission. Based on a study by Aulmann et al., the scoring performed on the operation day itself has a better prognostic value than the scores taken during admission (12). Nevertheless, based on the outcome in this study at three months after discharge, $33.3 \%$ had the mRS score of o with no symptoms at all, and $33.3 \%$ had the mRS score of 6 or had died. After an additional three months after discharge, three patients with mRS scores of 1 or 2 improved to the mRS score of $\mathrm{o}$. This improvement is mainly due to the aftercare of patients, which comprises intensive rehabilitation and physiotherapy.

\section{Conclusion}

The main characteristics of patients in Hospital Sungai Buloh were that they were relatively young (in their 40s) and mostly Malays without any known comorbidities. Although the majority had high Fisher grades, most had favourable outcomes post intervention. Data collected from Hospital Sungai Buloh may not be representative of the entire country's population. Therefore, a larger-scale data collection is necessary to create our own database to identify the risk factors that are exclusive to our country, if present.

\section{Acknowledgement}

I would like to thank first and foremost my supervisor; Mr Azmin Kass Rosman, who has been very helpful in guiding me through the whole process of completing this task and also giving me the courage to continue on with this journey despite the challenges and difficulties that arises.

To Professor Dato' Dr. Jafri Malin Abdullah, Associate Professor Zamzuri Idris, Dato' Dr, Abdul Rahman Izani Ghani and Dr Regunath Kandasamy, Neurosciences lecturers of Universiti Sains Malaysia, I thanked you all for encouraging us to produced more publications! 


\section{Conflict of Interest}

None

\section{Funds}

None

\section{Authors' Contribution}

Conception and design: AAG, SAMN, RK, AKR, ARIG Analysis and interpretation of the data: AAG, SAMN, RK, AKR, ARIG

Drafting of the article: AAG, SAMN, RK, AKR, ARIG

Critical revision of the article for important intellectual content: AAG, SAMN, RK, AKR, ARIG

Final approval of the article: AAG, SAMN, RK, AKR, ARIG

Provision of study materials or patients: AAG, SAMN, AKR

Statistical expertise: AAG, SAMN, RK, AKR, ARIG

Administrative, technical, or logistic support: AAG, SAMN, RK, AKR, ARIG

Collection and assembly of data: AAG, SAMN, AKR

\section{Correspondence}

Dr. Ailani Ab. Ghani

MB BCh BAO (NUI), Master of Surgery (Neurosurgery),

Universiti Sains Malaysia

Department of Neurosurgery,

Hospital Sungai Buloh, Jalan Hospital,

47000 Sungai Buloh,

Selangor, Malaysia

Tel: $+603-61454333$

Fax: +603-61454222

E-mail: ailaniabghani@yahoo.com

\section{References}

1. Mayberg MR, Batjer $\mathrm{HH}$, Dacey $\mathrm{R}$, Diringer M, Haley EC, Heros RC, et al. Guidelines for the management of aneurysmal subarachnoid hemorrhage. A statement for healthcare professionals from a special writing group of the Stroke Council, American Heart Association. Stroke; A Journal of Cerebral Circulation. 1994;25(11):2315-2328.

2. de Rooij NK, Linn FH, van der Plas JA, Algra A, Rinkel GJ. Incidence of subarachnoid haemorrhage: a systematic review with emphasis on region, age, gender and time trends. Journal of Neurology, Neurosurgery, and Psychiatry. 2007;78(12):1365-1372. http://dx. doi.org/10.1136/jnnp.2007.117655.
3. Chee CP. Subarachnoid haemorrhage, aneurysm and arteriovenous malformation: a review of Malaysian and South East Asian population. Neurol J Southest Asia. 1998;3:51-55.

4. Wong JS, Ng KH, Wong SH. Intracranial aneurysms in Sarawak General Hospital over a 30-month period. Journal of clinical neuroscience: Official journal of the Neurosurgical Society of Australasia. 2004;11(3):254-258. http://dx.doi.org/10.1016/ So967-5868(03)oo133-4.

5. Rinkel GJ, Djibuti M, Algra A, van Gijn J. Prevalence and risk of rupture of intracranial aneurysms: a systematic review. Stroke; a journal of cerebral circulation. 1998;29(1):251-256.

6. Samandouras G. Subarachnoid haemorrhage 1: diagnosis. The neurosurgeon's handbook. Oxford: Oxford University Press; 2010.

7. Ingall $\mathrm{T}$, Asplund $\mathrm{K}$, Mahonen $\mathrm{M}$, Bonita $\mathrm{R}$. A multinational comparison of subarachnoid hemorrhage epidemiology in the WHO MONICA stroke study. Stroke; a journal of cerebral circulation. 2000;31(5):1054-1061.

8. Wardlaw JM, White PM. The detection and management of unruptured intracranial aneurysms. Brain: A journal of neurology. 2000;123(Pt2):205-221.

9. Khurana VG, Mayer FB. Biology of cerebral blood vessels and blood flow. In: Le Roux PD, Winn HR, Newell DW, editors. Management of Cerebral Aneurysms. Pennsylvania, Philadelphia: WB Saunders Co; 2004:139-143.

10. Batjer HH, Chandler JP, Getch CC, Gravely L, Bendok BR. Intracranial aneurysm. In: Rengachary SS, Ellenbogen RG, editors. Principles of Neurosurgery. Edinburgh: Elsevier Mosby Publishing; 2005:215-239.

11. Navarro JEV. A proposed scoring system to screen for vasospasm following aneurysmal subarachnoid hemorrhage. Neurology Asia. 2007;12:7-11.

12. Aulmann C, Steudl WI, Feldmann U. Validation of the prognostic accuracy of neurosurgical admission scales after rupture of cerebral aneurysms. Zentralblatt fur Neurochirurgie. 1998;59(3):171-180. 